\title{
A Different Approach to the Management of Greater Trochanter Pain Syndrome
}

\begin{abstract}
Greater trochanter pain syndrome (GTPS), also known as trochanteric bursitis, is a regional pain syndrome that is frequently treated by physiotherapists in private practice or out-patient departments. It is classified as an overuse injury that could become chronic in nature and frequently co-exists with other pathologies.

This case study describes the treatment of a 61-year-old female with GTPS of her left hip. The aim was to evaluate the effectiveness of specific soft tissue mobilisation (SSTM) and eccentric strengthening of the Gluteus Medius (GM) muscle in treating this condition. Particular emphasis was placed on rehabilitation of lumbar spine control in order to improve proximal stability. A nother aim was to return the patient faster to her functional activities than had been reported in the literature.

The patient could return to her normal daily activities after four treatment sessions and was completely pain free after 12 weeks. This case study presents a different approach to the treatment of GTPS and proposes that GTPS may present in a similar manner to GM tendinosis. This phenomenon could therefore possibly explain the chronic nature of the condition.
\end{abstract}

KEYWORDS: GREATER TROCHANTER PAIN SYNDROME, GLUTEUS MEDIUS TENDINOSIS, SPECIFIC SOFT TISSUE MOBILIZATION, ECCENTRIC STRENGTHENING.

\section{INTRODUCTION}

Trochanteric bursitis is a common regional pain syndrome (Bird et al 2001, Jones and Erhard 1997, Kingzett-Taylor et al 1999, Paluska 2005, Shbeeb et al 1996). Although it is referred to as an inflammatory condition, the clinical signs of redness, local heat and swelling are not usually demonstrated (Bird et al 2001, Paluska 2005, Shbeeb et al 1996). The appropriate term for this condition has been suggested as Greater Trochanter Pain Syndrome (GTPS) (Bird et al 2001, Kingzett-Taylor et al 1999, Paluska 2005, Shbeeb et al 1996). This syndrome has been reported to typically affect soft tissue structures, specifically the Gluteus Medius (GM) tendon and the greater trochanter bursa (Bird et al 2001, Paluska 2005, Shbeeb et al 1996).

Repetitive microtrauma caused by the active use of the GM and Gluteus Minimus muscles, are often associated with GTPS (Bird et al 2001, Paluska 2005). This repetitive microtrauma leads to degenerative changes to the muscles, tendons and fibrous tissues (Jones and Erhard 1997, Shbeeb et al 1996). GM tendon pathology, such as tendinosis and tears, has been identified by means of MRI imaging (Bird et al 2001, KingzettTaylor et al 1999). Chung et al (1999) also found atrophy of the GM muscle. Women are four times more likely to be affected by GTPS, than men and the incidence peaks between 40 and 60 years of age (Bird et al 2001, Chung et al 1999, Kingzett-Taylor et al 1999, Lievense et al 2005, Paluska 2005, Shbeeb et al 1996).

Physiotherapy modalities such as ice, heat and diathermy are used to treat GTPS (Anderson et al 2001, Bird et al 2001, Jones and Erhard 1997, Lievense et al 2005, Paluska 2005, Shbeeb et al 1996). Correcting muscular imbalances, especially following a rehabilitation programme focussing on hip rotational strengthening, are advocated by a number of authors (Anderson et al 2001,
Johnston et al 1998, Paluska 2005). None of the reviewed literature mentioned the use of specific soft tissue mobilization (SSTM) and eccentric hip abductor strengthening as treatment modalities.

SSTM is an assessment and treatment approach, based on evidence that supports the importance of carefully applied movement to restore tensile strength and functional biomechanical properties in healing soft tissue (Hunter 1998). SSTM can be applied during the regeneration and remodelling phase of soft tissue healing (Hunter 1998). The aim would

\section{Correspondence to:}

Mr Corné van Rooy

PO Box 1706

Brackenfell

7561

Tel: (021) 981-4727 (w)

Fax: 0865544578

E-mail: cvanrooy@telkomsa.net 
be to increase collagen synthesis, reorientate collagen fibers and improve the visco-elastic response of the tissue (Hunter 1998). The application of SSTM to degenerative tissue may also produce an inflammatory response that initiates the healing process (Hunter 1998).

The healing process seems to be influenced positively by subjecting patients to a treatment programme that focused on eccentric strengthening of the tendon (Alfredson et al 1998, Jonsson et al 2005, Silbernagel et al 2001). These programmes included relatively high and progressive loads (Alfredson et al 1998, Jonsson and Alfredson 2005, Silbernagel et al 2001). It aided the healing process by remodelling tendon fibers and improving tensile strength (Alfredson et al 1998, Jonsson and Alfredson 2005, Silbernagel et al 2001, Svernlöv and Adolfson 2001).

This case study aimed to investigate the effects of SSTM and eccentric strengthening as treatment modalities in the rehabilitation of patients suffering from GTPS.

\section{CASE PRESENTATION}

The patient was a 61-year-old female. She was a healthy, independent grandmother. She participated in walking and water aerobics to maintain her fitness. She walked twice a week and did water aerobics 4-5 times per week. She participated in fun walks during the year.

\section{Presentation of symptoms}

The patient complained of left lateral thigh pain. She experienced the pain especially at night during left side lying (Figure 1). She was slightly aware of P1 during sitting (3/10 Visual analogue scale), moving from sitting to standing $(3 / 10$ VAS $)$ and descending stairs (5/10 VAS). Avoiding these movements decreased P1. P1 did not prevent her from doing her daily activities. In the morning she experienced P1 3/10 (VAS) when she got out of bed.

\section{Previous history}

She had no history of previous injuries in the hip region. She had a complete break in her exercise routine due to a laparoscopic right hemi-colectomy in
Figure 1: Area and description of pain.

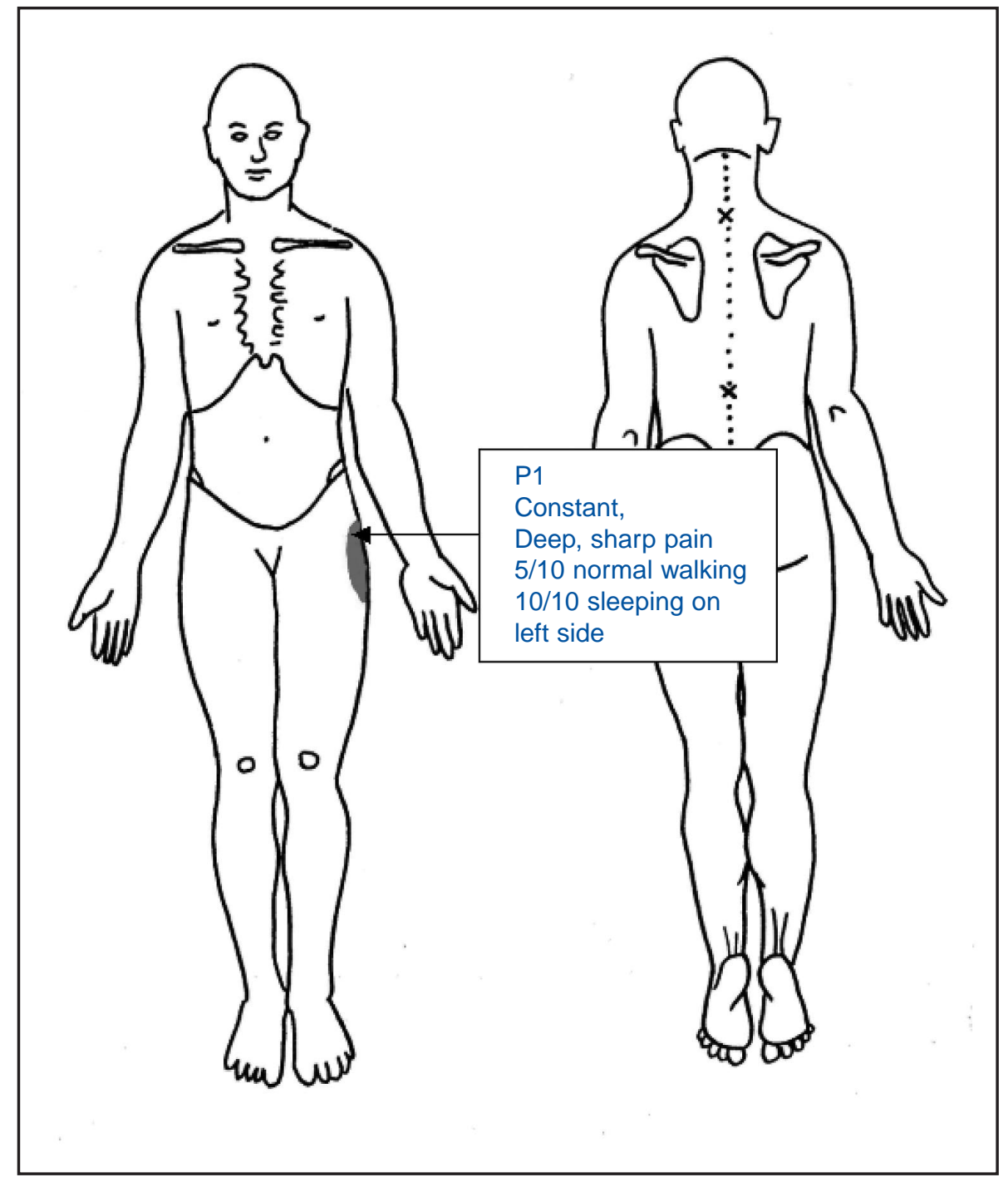

Figure 2: Movement diagram.

L (Muscular)

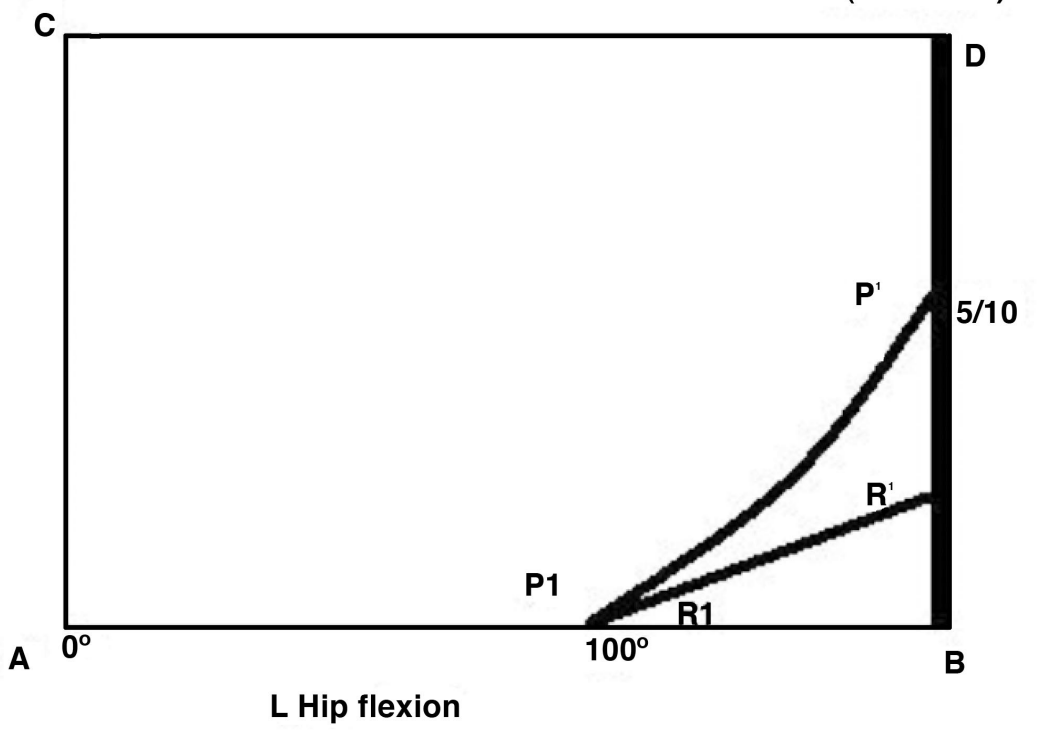




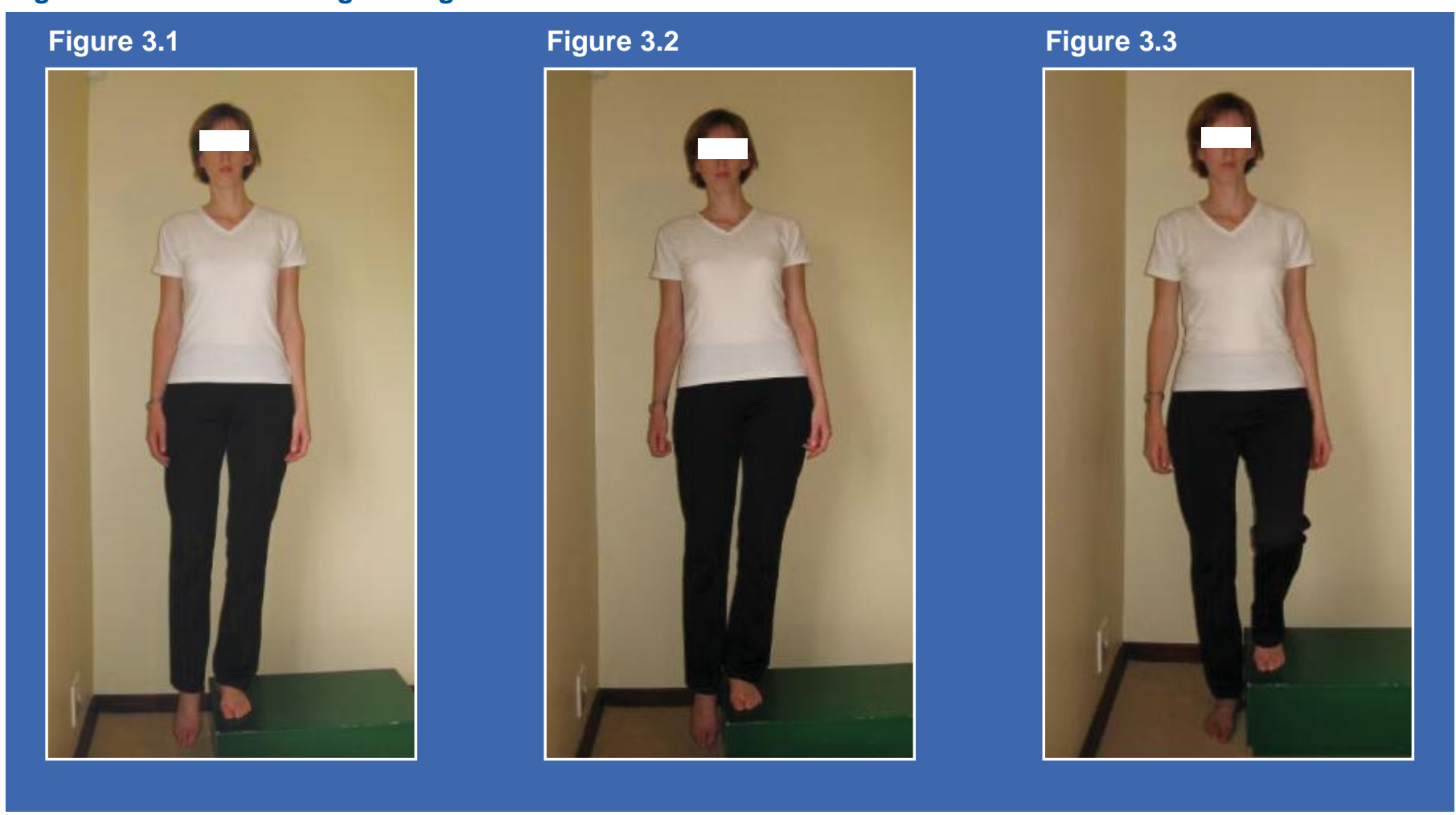

November 2006. Her first walk was two months after her surgery when she completed a $13 \mathrm{~km}$ walk on the beach. She experienced delayed onset muscle soreness in her thighs and calves the day thereafter. This happened two weeks prior to the onset of $\mathrm{P} 1$.

\section{Physical Examination}

The patient walked into the room unaided. No gait abnormalities were observed. She pointed to the left lateral thigh when asked to show the area of her pain (Figure 1). Squatting revealed normal range of movement (ROM) at the hip, knees and ankles, but she experienced pain 3/10 (VAS) in the P1 area. On active ROM testing of the hip, she experienced $\mathrm{P} 1$ as $3 / 10$ (VAS) through the range of left hip abduction. Passive left hip adduction reproduced her pain and slight resistance was felt towards the end of range with a muscular end feel. The pain increased gradually through to end range where it peaked at 5/10 (VAS). She also experienced P1 on passive hip flexion (Figure 2).

Muscle testing according to the Oxford scale revealed normal muscle strength of all hip and leg muscles, but she experienced pain on abduction $(5 / 10$
VAS). Isometric testing of abduction and flexion of the left hip reproduced $3 / 10$ pain (VAS) in her left lateral thigh. Her neurological examination was clear.

On soft tissue palpation she had tightness in her left GM and left gluteus maximus muscles, with extreme tenderness over the left trochanteric bursa. This reproduced $\mathrm{P} 1$.

\section{MANAGEMENT}

\section{Session 1}

The patient presented with GTPS during the regeneration phase of the healing process. A grade II postero-anterior (PA) force (according to Maitland's principles) was applied to the GM insertion at the greater tuberosity with the patient in right side lying. The stiff parts of the GM were also mobilised with SSTM in a transverse direction (Maitland 1999). She was instructed to refrain from her walking training, but was allowed to continue with water aerobics provided it did not increase her symptoms. The patient reported a subjective decrease in pain from 5/10 to 2/10 (VAS) with weight bearing on the affected leg. Passive hip flexion increased to $110^{\circ}$ with P1 3/10 (VAS).

\section{Session 2}

She reported that her night symptoms had decreased from $10 / 10$ to $5 / 10$ (VAS). She had 3/10 pain (VAS) during walking. The SSTM treatment was repeated and the grade was progressed to a grade III. The leg was also lowered into adduction (over the side of the bed) to gradually increase the tension on the hip abductors. Her pain decreased to $1 / 10$ (VAS) on re-evaluation of walking. Passive hip flexion increased to $120^{\circ}$ with P1 1/10 (VAS).

\section{Session 3}

The patient experienced $1 / 10$ pain (VAS) during weight bearing on the left leg and $2 / 10$ pain (VAS) while lying on the left side at night. The SSTM treatment was repeated as described in session 2. The patient was instructed in eccentric hip abduction as a home exercise.

The exercise was executed with the patient standing on her left leg with her foot on the edge of a step. She had to lower the right side of the pelvis in a slow controlled movement until her right foot was lowered below the edge of the step. Then she would take her body weight onto her right leg and bring her left leg down to ground level. She 
returned to the starting position by climbing up the step with her right leg. This emphasized the eccentric action of her hip abductors. She was given two sets of 10 repetitions once a day. Some degree of pain, up to 5/10 (VAS) was allowed during this exercise (Figure 3 ). She reported her walking to be pain free on re-evaluation after the session. Passive hip flexion was pain free.

\section{Session 4: Further avenues}

She did not report any change in her symptoms. The improvement seemed to have reached a plateau. She reported her pain as 1/10 (VAS) during walking and at night as 2/10 (VAS).

After specific questioning about her lower back, she mentioned that she did experience discomfort and pain in the lower lumbar spine at times, especially in the mornings upon waking. Her lumbar spine was then evaluated and revealed slight left sided pain in her lower lumbar spine with over pressure of lumbar extension. All other physiological movements were cleared with overpressure. On palpation the L4/5 facet joint on her left was painful with a grade III unilateral PA and some stiffness was felt in the joint. This accessory movement also reproduced P1 2/10 (VAS). The L4/5 facet joint was then mobilized with a grade 111 unilateral PA (Maitland 1999). This was done for 45 seconds and repeated three times (Maitland 1999). She reported that weight bearing during walking was then pain free.

Due to the symptoms reproduced from her lumbar spine, observation of her Transversus Abdominus (TrA) contraction in crook lying was evaluated. This revealed that she substituted with her external and internal obliques. She was then taught correct TrA isolation in crook lying. It was added to her rehabilitation exercise programme.

She was given permission to start walking again but only for short distances, e.g. 20 minutes on a level surface. She had to stop the walking if she became aware of any increase in her symptoms. One session per week was allowed initially, which she increased gradually over two to three weeks until she reached her normal walking programme.

\section{Session 5}

The patient experienced $1 / 10$ pain (VAS) on lumbar extension. This was mobilized as in session 4. SSTM was repeated as described in session 3 .

\section{Session 6}

She reported 1/10 pain (VAS) at night. She could lie on the left side without feeling the need to turn onto her right side. She did not experience any pain on weight bearing. Her stabilizing exercises were progressed to more dynamic exercises. These included lunges, bridging with her lower legs supported on a physio ball and flexing her hip, while maintaining a neutral spine in a seated position on a physio ball. She did 10 repetitions of every exercise twice per day.

\section{Session 7: Re-evaluation}

She did not experience any symptoms in her left lateral thigh or lumbar spine (twelve weeks after injury). Palpation of the left GM insertion at the greater trochanter bursa was completely pain free and the left L4/5 facet joint was cleared with Grade IV overpressure. She had returned to her normal walking routine and was discharged from physiotherapy. She continued with her strengthening exercises at least twice per week for six weeks to maintain her muscle strength. She was also informed that she should return for physiotherapy treatment if she noticed pain or discomfort in her left lateral thigh.

\section{DISCUSSION}

\section{GTPS - Nature of the Condition}

Literature sources have reported that GTPS is a chronic condition that leads to tendinosis of the GM muscle (Bird et al 2005). Applying SSTM to degenerative tissue may produce an inflammatory response that initiates healing (Hunter 1998). As the healing process continues to the regeneration phase, SSTM may promote functional collagen alignment and increase cross linkages between collagen fibers causing them to become more stable (Hunter 1998). The patient had a substantial reduction in the pain after two treatment sessions. She was able to return to her functional activity of walking after the fourth treatment.
SSTM of the GM seemed to be an effective technique to decrease pain and dysfunction.

\section{Co-existing conditions}

It was noted from the literature that GTPS very seldom exists as a condition on its own and often co-exists with other underlying conditions, such as lumbar facet joint pain and lumbar spine stiffness (Shbeeb et al 1996). Emphasis should be placed on an extensive interview covering all possible related areas. This will ensure that any underlying conditions are identified early and appropriate evaluation and treatment strategies are implemented to address these conditions effectively. The patient in this case study became pain free only after the appropriate treatment for her lumbar spine was performed.

\section{Previous medical intervention}

The laparascopic hemi-colectomy the patient underwent should also be considered. Although there were no major surgical incisions made in the abdominal muscles, the initial pain and discomfort after the surgery may have influenced the effective activation of $\operatorname{Tr} A$. Inactive TrA could have decreased the patient's lumbar spine stability when she returned to her exercise. Decreased spinal stability could possibly have led to overuse of the GM muscle as it would try to stabilize the pelvis during walking to compensate for decreased $\operatorname{Tr}$ A function.

\section{Management}

Successful management of GTPS depends greatly on dynamic movement control during activities of daily living, training and competitive involvement (Anderson et al 2001, Paluska 2005, Shbeeb et al 1996). Dynamic control of the hip, pelvis and lower lumbar spine is of special importance (Anderson et al 2001, Paluska 2005, Shbeeb et al 1996). The shorter recovery period noted with the patient in this case study, may be attributed to the treatment approach followed. This approach focused on improving lumbar spine stability and control as well as the eccentric strengthening of the GM muscle. Clinical trails have supported the use of eccentric strengthening as a treatment modality 
for tendinosis (Adolfson 2001, Jonsson et al 2005, Silbernagel et al 2001, Svernlöv and). This also proved to be the case with the patient.

\section{CONCLUSION}

This case study reported on the rehabilitation of a female walker who suffered from chronic greater trochanter pain syndrome. The underlying pathology of this condition may mimic the pathological changes noted in chronic tendon pathologies. This case study highlights that patients with this clinical presentation may benefit from GM eccentric training and SSTM mobilization rehabilitation strategies. Further research into the pathology and effectiveness of intervention strategies are required.

\section{REFERENCES}

Anderson K, Strickland SM, \& Warren R 2001 Hip and groin injuries in athletes. The American Journal of Sports Medicine 29: 521-533
Bird PA, Oakley SP, Shnier R, Kirkham BW 2001 Prospective evaluation of magnetic resonance imaging and physical examination findings in patients with greater trochanteric pain syndrome. Arthritis \& Rheumatism 44: 2138-2145

Chung CB, Robertson JE, Cho GJ, Vaughan LM, Copp SN, Resnick D 1999 Gluteus medius tendon tears and avulsive injuries in elderly women: Imaging findings in six patients. American Journal of Roentgenology 173: $351-353$

Hunter G 1994 Specific soft tissue mobilization in the treatment of soft tissue lesions. Physiotherapy 80: 15-21

Hunter G 1998 Specific soft tissue mobilization in the management of soft tissue dysfunction. Manual Therapy 3: 2-11

Jones DL, Erhard RE 1997 Case report: Diagnosis of trochanteric bursitis versus femoral neck stress fracture. Physical Therapy Journal 77: 58-67

Jonsson P, Alfredson H 2005 Superior results with eccentric compared to concentric quadriceps training in patients with jumper's knee: a prospective randomized study. British Journal of Sports Medicine 39: 847-850
Johnston CAM, Wiley JP, Lindsay DM, Wiseman DA 1998 Iliopsoas bursitis and tendonitis. Sports Medicine Journal 25: 271-283

Lievense A, Bierma-Zeinstra S, Schouten B, Bohnen A, Verhaar J, Koes B 2005 Prognosis of trochanteric pain in primary care. British Journal of General Practice 55: 199-204

Maitland GD 1999 Vertebral manipulation, 5th edn. pp96-97. Butterworth Heinemann, Oxford

Paluska SA 2005 An overview of hip injures in running. Sports Medicine 35: 991-1014

Shbeeb MI, Matteson EL 1996 Trochanteric bursitis (Greater Trochanter Pain Syndrome). Mayo Clinic Proceedings 71: 565-569

Silbernagel KG, Thomeé R, Thomeé P, Karlsson J 2001 Eccentric overload training for patients with chronic Achilles tendon pain - a randomised controlled study with reliability testing of the evaluation methods. Scandinavian Journal of Medicine \& Science in Sports 11: 197-206

Svernlöv B, Adolfson L 2001 Non-operative treatment regime including eccentric training for lateral humeral epicondylalgia. Scandinavian Journal of Medicine \& Science in Sports 11: 328-334 